\title{
Variedades de carozos (Chile y Cuyo 1700-1850)
}

\author{
Stone-fruit varieties (Chile and Cuyo 1700-1850) \\ Lacoste P. ${ }^{1}$, Yuri J.A. ${ }^{2}$, Aranda M. ${ }^{3}$, Castro A. ${ }^{4}$, Quinteros K., Solar M., Soto N., Chávez C.
}

\section{RESUMEN}

El estudio hace visibles las variedades tradicionales de carozos (durazneros, ciruelos, damascos, guindos y cerezos), cultivados en Chile y Cuyo entre 1700 y 1850. A partir del corpus documental de fondos notariales y judiciales de los archivos de Chile, San Juan y Mendoza se identificaron nueve variedades de duraznos, nueve de ciruelos, cuatro de damascos y dos de guindos. Asimismo, se han encontrado vinculaciones respecto a la apreciación de la cultura de la fruta entre la tradición musulmana de al-Andalus y la tradición iberoamericana.

Palabras clave: Agricultura tradicional, fruticultura, frutas de carozo, variedades.

\section{ABSTRACT}

The present study investigated the traditional stone (peach, plum, apricot, sour cherry and sweet cherry) fruit varieties grown in Chile and Cuyo (1700-1850). The documentation of the study was made from notary and judicial archives from Chile, San Juan and Mendoza. Nine varieties of peach, seven varieties of plum, four of apricot and two of sour cherry were identified. Also, important links were found concerning the appreciation of fruit culture between the Muslim traditions of al-Andalus in Spain and Latin-American traditions.

Key words: Traditional agriculture, fruit culture, stone-fruit, fruit variety.

El presente trabajo tiene como objetivo realizar un aporte desde la documentación histórica para el conocimiento de las especies frutales de carozo y sus variedades en Chile y Cuyo entre 1700 y 1850. El propósito es contribuir al proceso de identificación del origen, la identidad y las relaciones de las plantas, en forma complementaria a los estudios de secuencia de $\mathrm{ADN}$ que se realizan en los laboratorios, tal como han señalado los agrónomos que actualmente están trabajando en el tema (Ibáñez, 2011; Cabello Sáenz, 2011). La idea es, desde los métodos y fuentes propios de la historia, entregar información relevante sobre la evolución de las variedades en una de las principales regiones frutícolas de América Latina, en el marco de un proyecto mayor, que ya ha permitido dar a conocer resultados sobre variedades de uva (Lacoste et al., 2010) y de pomáceas (Lacoste et al., 2011).

Se estudiaron las jurisdicciones de La Serena, San Felipe, Santiago, San Fernando, Parral, Cauquenes, Mendoza y San Juan, desde 1700 hasta mediados del siglo XIX, a partir de registros notariales y judiciales obrantes en los fondos del Archivo Nacional de Santiago (AN), el Archivo Histórico de Mendoza (AHM), el Archivo General de la Provincia de San Juan (AGPSJ) y el Archivo del Poder Judicial de San Juan (APJSJ). En cada caso se han verificado datos de especie y variedad. Se han compulsado documentos originales inéditos, principalmente inventarios de bienes, testamentos, cartas de dote. El trabajo de archivo ha permitido identificar 93.119 plantas de carozos, con notable liderazgo de los durazneros:

1 Profesor Titular de la Universidad de Santiago de Chile (USACH). Instituto IDEA. Román Díaz 89, Providencia, Santiago de Chile. Teléfono (56-2) 7181360. E-mail: pablo.lacoste@usach.cl

2 Director del Centro de Pomáceas de la Universidad de Talca. 2 Norte 685. Talca, Chile. Teléfono (56-71) 200200. E-mail: ayuri@utalca.cl

3 Post doctoranda USACH. Instituto IDEA. Román Díaz 89, Providencia. Santiago de Chile. Teléfono (56-2) 7181360. E-mail: marcela.aranda06@gmail.com

4 Profesora de la Universidad Católica Silva Henríquez. General Jofré 462. Santiago de Chile. Chile. Teléfono (56-2) 4601100. E-mail: castrosancarlos@yahoo.com.mx 

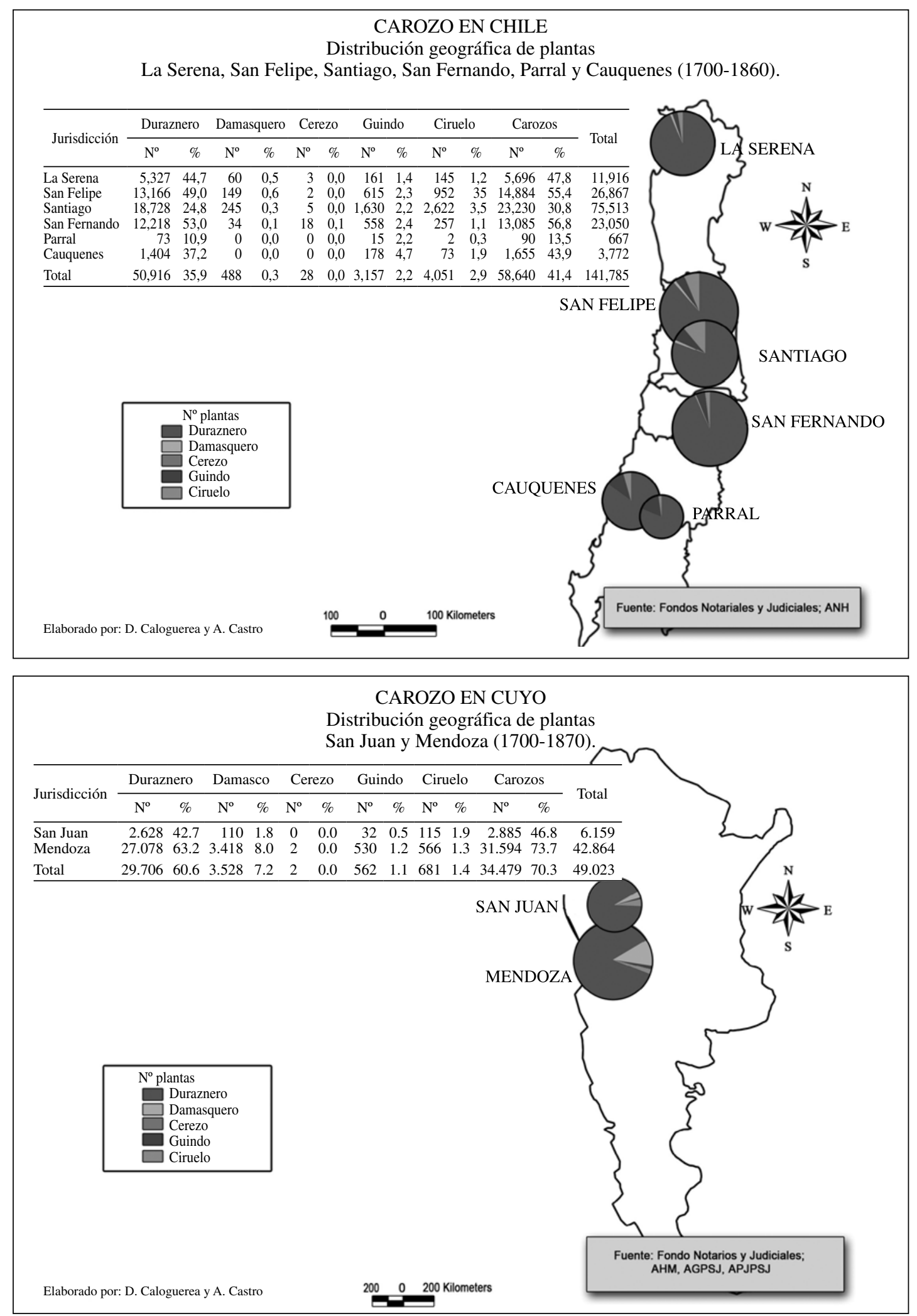

Figuras 1-2. Distribución geográfica de carozos en Chile entre 1700 y 1860, expresada en número de plantas y porcentaje. 
estos ascendían a 80.622 ejemplares ( $86 \%$ del total). También había 4.732 ciruelos (5\%), 3.719 guindos (4\%), 4.016 damascos $(4,3 \%)$ y 30 cerezos (Figuras 1 y 2). A partir de este corpus documental se examinaron las variedades. Las fuentes aportaron información de variedades de 403 plantas de carozo, incluyendo 129 durazneros, 221 ciruelos, 39 damascos y 14 guindos. Sobre la base de esta información, se distinguen las variedades cultivadas, atendiendo datos como lugar, fecha y cantidad de ejemplares de cada registro.

La tradición de identificar y clasificar las variedades de frutales tuvo un punto de inflexión con el aporte de los agrónomos y botánicos andalusíes en el sur de la península ibérica. A través de sus manuales ha sido posible conocer el notable grado de avance que alcanzó la cultura musulmana con respecto al conocimiento de las plantas frutales en general y sus variedades en particular. Dentro del duraznero se identifican dos grandes grupos: a) con pelusa y b) $\sin$ pelusa. El primer grupo, a su vez, se subdivide en dos grupos: a1) el durazno que se separa fácilmente de su hueso, y a2) el que no se raja -o cerrado. El segundo grupo lo forma el melocotón de la India, liso y sin pelusa, del cual "la gente dice que es un injerto con ciruelo", el que, a su vez, se divide en tres: b1) calvo; b2) de invierno y b3) luffah (meloncillo). "Esta última especie de melocotón, liso y de color rojo, al que los egipcios llaman 'florido' (zahrawi), es la mejor, muy aromática, jugosa y más penetrante y un sabor más agradable, aunque menos dulce que la velluda, y también es menos húmeda" (Carabaza Bravo et al., 2004: 166).

Por su parte, para los agrónomos andalusíes el ciruelo se clasifica en "cultivado y silvestre; de ciruela amarilla, negra o blanca, con muchas variedades de estos dos últimos tipos, tantas que apenas existe parecido entre algunas, salvo el nombre del género que es común. La blanca puede ser grande o pequeña; la negra, silvestre o cultivada, y dentro de ella hay tres variedades, de color más o menos intenso, dependiendo del punto de maduración. Se distinguen varias: una variedad negra y grande, conocida por tari (fresca, jugosa); otra negra, muy común, que se da en invierno, llamada igual que la anterior; otra de color negro verdoso, gorda, llamada azyar; otra blanca, amarilla y roja, y finalmente las conocidas como qarmisi (carmesí) y sayhi (estriada)" (Carabaza Bravo, 2004: 158). Con respecto al damasco, "atendiendo al tamaño del fruto, hay dos: menudo o gordo" (Carabaza Bravo, 2004: 146).
Junto con las variedades específicas, los agrónomos y botánicos de al-Andalus entregaron una serie de criterios para distinguir una de otra, los cuales servirían después como matriz para construir el sistema de variedades de Chile y Cuyo: a) color de la fruta: ciruelos amarillo, blanco y negro; b) tamaño de la fruta: albaricoque menudo y gordo; ciruelo grande y pequeño; c) ciclo de cosecha: ciruelo de invierno, durazno de invierno; d) textura: durazno peludo o calvo; e) origen geográfico: durazno de la India, ciruelo de España.

Estos criterios fueron aplicados, siglos después, por los fruticultores chilenos para distinguir, identificar y clasificar las variedades de frutas de carozo. Tal como lo revelan las fuentes documentales compulsadas, en Chile y Cuyo se cultivaban nueve variedades de duraznero, nueve de ciruelo, cuatro de damasco y dos de guindo. Los Cuadros 1 , 2, 3 y 4 entregan información detallada al respecto, indicando lugar, año y cantidad de ejemplares de cada registro. Evidentemente, la distribución de estas variedades no era uniforme ni en el tiempo ni en el espacio examinado. También había diferentes recurrencias: algunas eran muy frecuentes y otras muy escasas. Esas asimetrías pueden examinarse en los cuadros ${ }^{1}$.

A la luz de las clasificaciones andalusíes, se pueden detectar algunos cambios y continuidades, pues las variedades de carozos americanos se agrupaban de acuerdo a los siguientes criterios: a) color y forma de la fruta: ciruelo blanco, ciruelo negro; ciruelo invernizo morado; damasco amarillo, damasco blanco; durazno pinto; ciruelo racimo; durazno abollado; b) sabor: durazno dulce; c) origen geográfico: ciruelo de España; damasco de España; durazno Montuero ${ }^{2}$; guindo Bella Vista (única denominación de origen chilena); d) semejanza de su fruta con la de otras plantas: durazno albaricoque, durazno manzano, durazno guindo y durazno almendruco; guindo cerezo; e) ciclo de cosecha: ciruelo invernizo; ciruelo invernizo morado; f) forma de cultivo: durazno montuero ${ }^{3}$; g) otro: durazno San José, durazno de la Virgen; ciruelo de La Purísima; damasco apricot; ciruelo culenar.

Para comprender mejor los datos de inventarios conviene contextualizarlos con las descripciones de los cronistas, cuyos detalles enriquecen la interpretación. Gómez de Vidaurre (1780) aseguró que se cultivaban más de doce variedades distintas de durazneros en Chile. No entrega mayores datos sobre cada una, pero llama la atención sobre dos frutas 
Cuadro 1. Variedades de duraznos en Chile y Cuyo (1777-1847).

\begin{tabular}{|c|c|c|c|c|c|c|c|c|c|c|c|c|}
\hline Localidad & Año & Fruticultor & Alb & Alm & Abu & Dul & Gui & Ма & Mo & SJ & Vir & Total \\
\hline San Juan & 1777 & Juana Balda & & & & 1 & & & & & & 1 \\
\hline San Fernando & 1786 & Ventura Maturana & & & & & & 16 & & & & 16 \\
\hline San Fernando & 1788 & Dionisio Armara & & & & & & & 100 & & & 100 \\
\hline San Fernando & 1812 & Rosa Quinteros & 2 & & & & & & & & 4 & 6 \\
\hline San Fernando & 1819 & Pascuala Serpa & & & & & $\mathrm{S} / \mathrm{D}$ & & & & & S/D \\
\hline San Juan & 1826 & José Javier Jofré & & & 1 & & & & & 1 & & 2 \\
\hline Mendoza & 1827 & Juan Jordán y Rosa Villegas & & & & & & & & 1 & 1 & 2 \\
\hline Mendoza & 1830 & Francisco Coria & & & & & & & & 1 & & 1 \\
\hline Mendoza & 1847 & $\begin{array}{l}\text { José Valderrama y María de } \\
\text { los Ángeles Saballa }\end{array}$ & & 1 & & & & & & & & 1 \\
\hline Total & & & 2 & 1 & 1 & 1 & $\mathrm{~S} / \mathrm{D}$ & 16 & 100 & 3 & 5 & 129 \\
\hline
\end{tabular}

Fuentes: Fondos notariales y judiciales (AN, AHM, AGPSJ, APJSJ).

Referencias: ALB: Durazno Albaricoque; ALM: Durazno Almendruco; ABU: Durazno Abollado; DUL: Durazno Dulce; GUI: Durazno Guindo; MA: Durazno Manzano; MO: Durazno Montuero; SJ: Durazno de San José; VIR: Durazno de la Virgen.

Cuadro 2. Variedades de ciruelos en Chile y Cuyo (1708-1863).

\begin{tabular}{|c|c|c|c|c|c|c|c|c|c|c|c|c|}
\hline Localidad & Año & Fruticultor & $\mathrm{Cul}$ & Esp & Neg & Rac & Inv & LPur & Bla & Pin. & Inv. M. & Total \\
\hline San Felipe & 1708 & Francisco Herrera & & & & 10 & & & & & & 10 \\
\hline Santiago & 1740 & María de Soto & & & & 2 & & & & & & 2 \\
\hline Santiago & 1740 & Marcela Paredes & & & & 1 & & & & & & 1 \\
\hline Santiago & 1742 & Francisco Calbacho & & & & 2 & & & & & & 2 \\
\hline Santiago & 1745 & Francisco Laguna & & & & 3 & & & & & & 3 \\
\hline Santiago & 1763 & Estancia Maipú & & & & 2 & & & & & & 2 \\
\hline Santiago & $1777-86$ & Ángela y Dorotea Silva & & & & 9 & & & & & & 9 \\
\hline San Felipe & 1786 & Gregorio Silva & & & & 1 & & & & & & 1 \\
\hline San Fernando & 1812 & Manuel Gálvez & & 1 & & & & & & & & 1 \\
\hline San Fernando & 1812 & Miguel Román & & 5 & & & & & & & & 5 \\
\hline Santiago & 1813 & Alberto Aranda & & 14 & & & & & & & & 14 \\
\hline Santiago & 1814 & M. del Tránsito Hurtado & & 29 & & & & & & & & 29 \\
\hline San Fernando & 1819 & Pascuaza Serpa y J. Ibarra & $\mathrm{S} / \mathrm{D}$ & & & & & & & & & $\mathrm{S} / \mathrm{D}$ \\
\hline San Fernando & 1819 & Manuel Porras & & 7 & & & & & & & & 7 \\
\hline San Fernando & 1824 & Juan Tapia & & 2 & & & & & & & & 2 \\
\hline San Fernando & 1826 & Josefa Madrid & & 2 & & & & & & & & 2 \\
\hline San Fernando & 1827 & Tomás Moya & & 2 & & & & & & & & 2 \\
\hline San Felipe & 1833 & José M. Segura & & 4 & & & & 4 & & & & 8 \\
\hline Santiago & 1838 & Antonio Aranguiz & & & 10 & & & & & & & 10 \\
\hline San Felipe & 1838 & Constanza Muñoz & & & & & & 20 & & & & 20 \\
\hline San Felipe & 1838 & José Mansilla & & & & & & & 1 & & & 1 \\
\hline San Felipe & 1839 & Ignacio Traslaviña & & 1 & & & & & & & & 1 \\
\hline Mendoza & 1863 & José M. Videla & & 67 & & & 3 & & & & & 70 \\
\hline Mendoza & 1842 & Margarita Medina de Pescara & & & & & & & & 2 & & 2 \\
\hline Mendoza & 1848 & María Lorenza Moyano de Maza & & & & & & & 2 & & & 2 \\
\hline Mendoza & 1849 & María Videla de Stela & & & & & & & 1 & & & 1 \\
\hline Mendoza & 1850 & Jacinto Godoy & & & & & & & & & 14 & 14 \\
\hline Total & & & S/D & 134 & 10 & 30 & 3 & 24 & 4 & 2 & 14 & 221 \\
\hline
\end{tabular}

Fuentes: Fondos notariales y judiciales (AN y AHM).

Referencias: CUL: Ciruelo Culenar; ESP: Ciruelo de España; NEG: Ciruelo Negro; RAC: Ciruelo de Racimo; INV: Ciruelo Inverniso; LPUR: Ciruelo de La Purísima; BLA: Ciruelo Blanco; PIN: Ciruelo Pinto. INV. M. (Invernizo Morado). 
Cuadro 3. Variedades de damascos en Chile y Cuyo (1812-1863).

\begin{tabular}{|c|c|c|c|c|c|c|c|}
\hline Localidad & Año & Fruticultor & España & Amarillo & Blanco & Apricot & Total \\
\hline San Fernando & 1812 & Miguel Román & 2 & & & & 2 \\
\hline Santiago & 1834 & Vicente Falcón y Carlos Solas de Navarrete & & 12 & 12 & & 24 \\
\hline Santiago & 1848 & Fermín Falcato e Ignacio Barahona & & 1 & & & 1 \\
\hline Mendoza & 1850 & Jacinto Godoy & & & 5 & & 5 \\
\hline Mendoza & 1863 & José M. Videla y Magdalena Correa de Videla & & & & 7 & 7 \\
\hline Total & & & 2 & 13 & 17 & 7 & 39 \\
\hline
\end{tabular}

Fuentes: Fondos notariales y judiciales (AN y AHM).

Cuadro 4. Variedades de guindos en Chile y Cuyo (1848-1863).

\begin{tabular}{lclcrr}
\hline Localidad & Año & Fruticultor & Cerezo & Bella Vista & Total \\
\hline Santiago & 1848 & José Ignacio Eyzaguirre & & 6 & 6 \\
Mendoza & 1863 & José María Videla y Magdalena Correa de Videla & 8 & 8 & 6 \\
Total & & & 8 & 6 & 14 \\
\hline
\end{tabular}

Fuentes: Fondos notariales y judiciales (AN y AHM).

distintas que daba la misma planta: los albérchigos y los almendrucos. Los duraznos albérchigos son frutos de árboles que "después de haber dado el mes de febrero de estos gruesos, se cargan otra vez para dar en abril otros pequeños, semejantes por la figura y por el grueso a las almendras, por lo que los llaman almendrucos. Estos no son menos dulces y gustosos que los primeros, y son más estimados para pasarlos por almíbar".

El durazno albérchigo, identificado en Chile a fines del siglo XVIII, era una variedad importante, que mantuvo su vigencia como una de las cuatro más relevantes, hasta comienzos del siglo XX. Al menos así se desprende de los escritos de D'Aygalliers, profesor de la escuela de agricultura de Oraison, autor de una obra traducida al español e incluida en la "Pequeña Enciclopedia de Agricultura". Este autor consideraba, fundamentalmente, cuatro variedades de duraznos: melocotones, pavías, albérchigos y abridores. El tercero de ellos se caracterizaba por tener "fruto liso, jugoso y carne adherida al hueso" (D’Aygalliers, 1904). En cierta forma, el durazno albérchigo podría asimilarse a la primera variedad identificada por los botánicos andalusíes.

Si Gómez de Vidaurre indicaba la existencia de doce variedades de durazneros, para Pérez García (1810) había catorce, de las cuales no mencionó nombres específicos. La principal diferencia entre una y otra la halló en el tiempo de maduración. De acuerdo a sus observaciones, esta amplia gama de variedades hacía posible que hubiera distintos tiempos de cosecha, con lo cual se prolongaba la temporada de los duraznos en Chile, "empezando en Navidad y acabando en junio". Poco después se elaboró otro informe, según el cual no había diferencias de sabor entre los duraznos, pero sí existía variación con respecto a la temperatura: "ciertas clases de duraznos se consideran por ejemplo como cálidos y otros como fríos" (Bladh, 1951).

A comienzos del siglo XIX, Louis Née (1793), el científico francés de la expedición de Malaspina, tuvo oportunidad de recorrer el territorio, identificar y clasificar los duraznos. En su informe mencionó la coexistencia de distintos "frutos de duraznos, unos de San José, otros de la Virgen, y éstos son pelados, otros llamados melocotones, en esta especie se incluyen abollados, mestizos, priscos, y éstos son de carne amarilla; priscos; albérchigos picudos; cambayes. Mas hay pelados priscos y con pelo; blancos y melocotones, esto es, duraznos, etc.".

$\mathrm{El}$ aporte de los cronistas permite interpretar mejor los datos de los inventarios de bienes y su correspondencia con las clasificaciones de los agrónomos andalusíes. Dentro de este esquema, como se recordará, se habían distinguido duraznos por su textura (peludos o pelados); a su vez, el durazno peludo podía ser fácilmente separable del carozo o estar cerrado; en tanto que el durazno pelado era llamado “de la India”. Cruzada esa clasificación con las variedades de Chile, se puede señalar que esas variedades coinciden con el durazno almendruco y el de la Virgen, respectivamente. Paralelamente, las observaciones de los cronistas señalan que la variedad 
de plantas de carozo era mayor a la que registraron los notarios en los inventarios de bienes.

\section{Conclusión}

Entre comienzos del siglo XVIII y hasta mediados del XIX la fruticultura chilena alcanzó una interesante diversidad de especies de carozos. Se llegaron a identificar nueve variedades de duraznero, nueve de ciruelo, cuatro de damasco y dos de guindo. Por las denominaciones utilizadas, es posible interpretar que hubo un lazo entre alAndalus y la fruticultura de Chile, pues los criterios aplicados para clasificar las variedades en ambos espacios geográficos exhiben varios puntos en común. La amplitud de las fuentes compulsadas -registros notariales y judiciales del Reino de Chile, testimonios de los cronistas, tratados de botánica, agricultura y fruticultura- permite complementar y profundizar esta información, sobre todo porque si bien los notarios americanos no disponían de conocimientos especializados para detectar las variedades, utilizaron términos y criterios que recogieron de usos y prácticas frutícolas de los pobladores hispanocriollos.

A pesar del desencuentro político y militar entre moros y cristianos, los conocimientos y la cultura de la apreciación de la fruta, introducida y difundida por los musulmanes en la península ibérica, se transmitió al pueblo español, el cual, a su vez, la trasladó al Reino de Chile, donde se desarrolló una rica fruticultura, caracterizada por la diversidad de cultivares.

\section{Agradecimientos}

Este trabajo fue financiado por el proyecto FONDECYT 1080210. Mario Solar, Katherine Quinteros, Cristian Chávez y Natalia Soto son ayudantes de dicho proyecto.

\section{Literatura Citada}

Agustín, M.

(1617) 1722 Libro de los secretos de la agricultura, casa de campo y pastoril. Barcelona, Imprenta Juan Piferrer.

Agüero, C. et al.

2003 "Identity and Parentage of Torrontés Cultivars in Argentina", American Journal of Enology and Viticulture, 54 (California), American Society for Enology and Viticulture, pp. 318-321.

Alonso de Herrera, G.

(1513) 1818 Agricultura general. Corregida según el texto original de la primera edición publicada en 1513 por el mismo autor y adicionada por la Real Sociedad Económica Matritense. Madrid, Imprenta Real.

Bladh, Carl E.

1951 La República de Chile: 1821-1851. Sociedad Chilena de Historia y Geografía. Santiago de Chile, Ed. Universitaria.

Cabello Sáez de Santa María, F.

2011 "Las variedades de vid cultivadas en la península ibérica a través de los libros de agricultura y ampelografía". I Conferencia Internacional "Patrimonio Cultural de la Vid y el Vino”, Almendralejo, Badajoz, 8 al 11 de febrero 2011.

Carabaza Bravo, J.M.; García Sánchez, E.; Hernández, E. y Jiménez Ramírez, A.

2004 Árboles y arbustos de Al-andalus. Madrid, CSIC.

D' Aygalliers, $\mathrm{P}$.

1904 Los árboles frutales y la viña. № 10 de la Colección de la Pequeña Enciclopedia de la Agricultura. Madrid, Ed. de Baillo-Bailliere é hijos.

Gómez de Vidaurre, F.

(1780) 1889 Historia geográfica natural y civil de Chile. Santiago de Chile, Ed. Ercilla.

Ibáñez, J.; De Andrés, M.T.; Zinelbidine, L.H.; Cabezas, J.A Gafario, L; Muñoz, G; Cabello, F; Martínez, Zapater, J.M.
2011 "Estudio de parentesco de variedades de vid mediante marcadores de ADN". I Conferencia Internacional "Patrimonio Cultural de la Vid y el Vino", Almendralejo, Badajoz, 8 al 11 de febrero 2011.

Lacoste, P.; Yuri, J.A.; Aranda, M.; Castro, A.; Quinteros, K.; Solar, M.; Soto, N.

2011 "Variedades de Pomáceas en Chile y Cuyo (1700-1850)", IDESIA 29, 1 (Arica), pp. 91-97.

Lacoste, P.; Yuri, J.A.; Aranda, M.; Castro, A.; Quinteros, K.; Solar, M.; Soto, N.; Gaete, J.; Rivas, J.

2010 "Variedades de uva en Chile y Argentina (1550-1850): genealogía del torrontés", Mundo Agrario 20: 1 (La Plata). http://www.mundoagrario.unlp.edu.ar/numeros/ no-20-1er-sem-2010/variedades-de-uva-en-chile-y-argentina-1550-1850-genealogia-del-torrontes <http://www. mundoagrario.unlp.edu.ar/numeros/no-20-1er-sem-2010/ variedades-de-uva-en-chile-y-argentina-1550-1850genealogia-del-torrontes>

Neé, L.

(1793) 2004 "Diario de viaje desde Talcahuano hasta Santiago de Chile,Concepción y Penco. Diciembre 16 de 1793”, en Sagredo Baeza, Rafael y José Ignacio González Leiva (2004), La expedición Malaspina en la frontera austral del imperio español. Santiago de Chile, Ed. Universitaria. Centro de Investigaciones Diego Barros Arana, pp. 827-870.

Pérez García, José A.

(1810) 1900 Historia de Chile. Tomo I. Colección de Historiadores de Chile y Documentos relativos a la Historia Nacional, tomo XXII. Santiago de Chile, Imprenta Elzeviriana.

Poeppig, Edward

(1726) 1960 Un testigo en la alborada (1826-1829). Santiago de Chile, Ed. Zig-Zag. 


\section{Notas}

1 Los Durazneros Montueros (100 plantas) representan el $24 \%$ del total (Cuadro 1). Le siguen los ciruelos, repartidos entre los Ciruelos de España (96 plantas), de Racimo (30) y de La Purísima (24), que constituyen el 54\%, el 16,4\% y el $13 \%$ del total, respectivamente (Cuadro 2). En cambio, el Damasco presenta una frecuencia intermedia pues Amarillos (13 plantas) y Blancos (17 plantas) suman el 35 y $46 \%$ del total, respectivamente (Cuadro 3 ). El número más bajo corresponde a los Guindos Cerezos (8 plantas) y significa el $57 \%$ del total de esta especie (Cuadro 4). También es posible leer los tiempos de cultivo de las variedades y con diferencias entre una y otra jurisdicción. En líneas generales, los carozos aparecieron primero en Chile, durante el siglo XVIII, sobre todo algunas variedades de durazno (dulces, manzanos y montueros) y ciruelo (de racimo). Desde la primera década del siglo XIX aparece el resto de las variedades, incluyendo damascos (de España y otros) y guindos (Bella Vista) al promediar el siglo. Los primeros registros de Cuyo datan de la tercera década del siglo XIX.

2 No ha sido posible definir con certeza el origen del nombre de la variedad "durazno montuero". Una posibilidad es que sea un origen geográfico referido a la localidad de Montuero (Castilla León).

3 La expresión "durazno montuero", además de la mencionada referencia geográfica, puede guardar relación con el lugar de cultivo (monte) por oposición a su alternativa (huerto). Los textos andalusíes distinguían entre planta frutal de huerto (hortense) y de monte (montesina). Posiblemente la denominación "durazno montuero" sea una derivación de una variedad que, en sus orígenes, se cultivaba en montes y no en huertos. 